ARTICLE OPEN

\title{
Large Josephson current in Weyl nodal loop semimetals due to odd-frequency superconductivity
}

\author{
Fariborz Parhizgar $\mathbb{D}^{1 凶}$ and Annica M. Black-Schaffer $\mathbb{D}^{1 凶}$
}

Weyl nodal loop semimetals (WNLs) host a closed nodal line loop Fermi surface in the bulk, protected zero-energy flat band, or drumhead, surface states, and strong spin-polarization. The large density of states of the drumhead states makes WNL semimetals exceedingly prone to electronic ordering. At the same time, the spin-polarization naively prevents conventional superconductivity due to its spin-singlet nature. Here we show the complete opposite: WNLs are extremely promising materials for superconducting Josephson junctions, entirely due to odd-frequency superconductivity. By sandwiching a WNL between two conventional superconductors we theoretically demonstrate the presence of very large Josephson currents, even up to orders of magnitude larger than for normal metals. The large currents are generated both by an efficient transformation of spin-singlet pairs into oddfrequency spin-triplet pairing by the Weyl dispersion and the drumhead states ensuring exceptionally proximity effect. As a result, WNL Josephson junctions offer unique possibilities for detecting and exploring odd-frequency superconductivity.

npj Quantum Materials (2020)5:42; https://doi.org/10.1038/s41535-020-0244-2

\section{INTRODUCTION}

In-between a conventional metal with its two-dimensional Fermi surface and a Weyl semimetal with its OD Fermi points, we find the Weyl and Dirac nodal loop semimetals (WNLs and DNLs), which have 1D nodal loop Fermi surfaces ${ }^{1,2}$. Multiple such materials have recently been both proposed ${ }^{3-11}$ and experimentally observed in compounds such as $\mathrm{PbTaSe}_{2}, \mathrm{ZrSiS}_{2} \mathrm{Ca}_{3} \mathrm{P}_{2}$, and $\mathrm{CaAgAs}^{12-15}$. Away from the nodal loop Fermi surface the dispersion is Weyl-like, completely locking the electron momentum to the electron orbital (for DNLs) or spin (for WNLs) degrees of freedom. As a result, the nodal loop in DNLs possesses a fourfold degeneracy, while WNLs break spin degeneracy and thus have only a twofold degenerate loop (crossing non-spin-degenerate valence and conduction bands). While the explicitly broken spin degeneracy have so far made the experimental realization of WNLs more demanding, there already exists candidate WNLs. For example, $\mathrm{HgCr}_{2} \mathrm{Se}_{4}$ was recently shown using ab initio calculations to be a $\mathrm{WNL}^{8,9}$ and experimental probes have also been proposed for how to easily detect the spin polarization ${ }^{10}$. Moreover, spin polarization has experimentally been found in $\mathrm{PbTaSe}_{2}$, making it a likely WNL candidate $^{12}$.

The nodal loop Fermi surface results in drumhead surface states at zero energy, whose area is set by the projection of the nodal loop on the surface plane ${ }^{16-18}$. The origin of this surface state is thus similar to that of the Weyl semimetals, but there only 1D surface arcs are formed due to the lower dimensionality of the bulk Fermi surface. The flat band dispersion of the drumhead states results in a large peak in the surface density of states (DOS). As a result, the surfaces become extremely prone to electronic ordering, including superconductivity which has already been discussed as a possibility for systems with surface flat bands ${ }^{19-21}$. In the bulk of WNLs, a 3D chiral superconducting state has also already been proposed based on both symmetry analysis ${ }^{22}$ and renormalization group calculations ${ }^{23}$. However, the strong spin polarization of WNLs, and in some cases even a complete spin polarization of the drumhead surface states, has been assumed to prohibit any spin-singlet superconductivity, and in particular including proximity effect from conventional superconductors $(\mathrm{SCs})^{24}$

The incompatibility of spin polarization and conventional (spin singlet, $s$-wave) superconductivity has actually been remedied in a few other cases by generating the exotic state of odd-frequency superconductivity ${ }^{25-27}$. Odd-frequency Cooper pairs are odd under the exchange of the relative time coordinate between the two electrons forming the pair, in contrast to the conventional equal-time pairing. As a consequence, odd-frequency pairing allows the common s-wave superconducting state to have spintriplet symmetry and still satisfy the necessary fermionic nature of superconductivity. In this way, odd-frequency spin-triplet pairing has been evoked to explain the long-ranged superconducting proximity effect measured in superconducting-ferromagnet junctions ${ }^{26,28,29}$, but odd-frequency superconductivity has also been found in nonmagnetic superconducting junctions ${ }^{30}$, as well as in bulk multiband $\mathrm{SCs}^{31}$ and driven systems ${ }^{32}$. As equal-time expectation values vanish for odd-frequency superconductivity, it becomes easily a hidden order and direct detection is notoriously hard. Still, odd-frequency superconductivity has been shown to impact physical properties ranging from the Meissner ${ }^{33-38}$ and Kerr effects ${ }^{39,40}$, to the existence of a finite supercurrent in half-metal (HM) Josephson junctions ${ }^{28,41-43}$. In the last case, the full spin polarization of the HM completely prohibits spin-singlet superconductivity, but Josephson effect has still been shown to be present in HMs with spin-active interfaces due to the creation of odd-frequency equal-spin-triplet pairing ${ }^{43}$.

In this work, we study a Josephson junction constructed by sandwiching a WNL between two conventional SCs, as shown schematically in Fig. 1a. Despite the strong spin polarization, which naively suppresses any proximity-induced superconductivity and thus Josephson effect, we find a huge Josephson current, even orders of magnitude larger than in normal metal (NM) and $\mathrm{HM}$ junctions. We first show how the spin-orbital Weyl interaction in WNLs results in a very efficient creation of equal-spin-triplet Cooper pairs, mimicking the spin arrangement in the normal state. It is these equal-spin pairs that carry the Josephson current, which

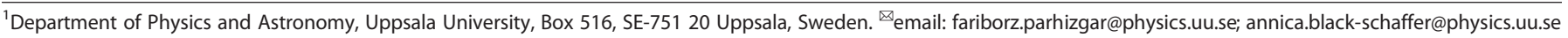




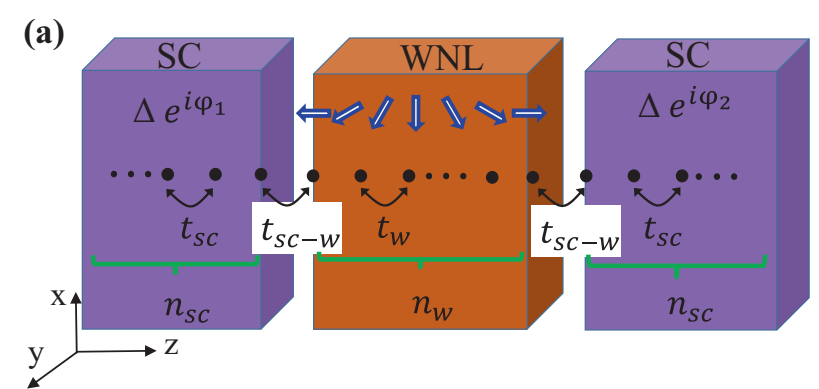

(b)

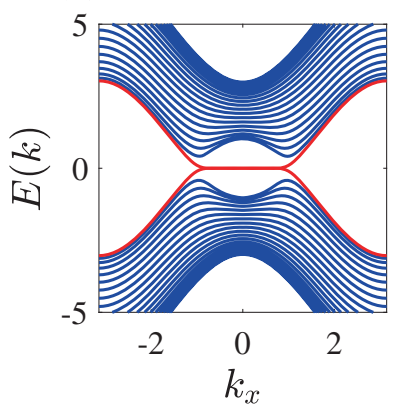

(c)

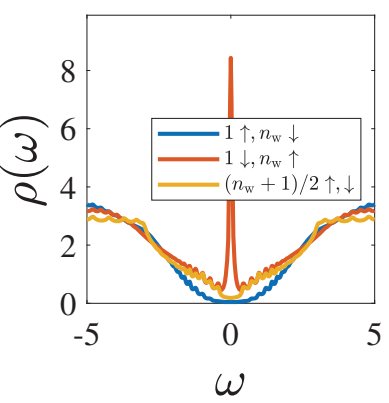

Fig. 1 WNL Josephson junction. a Schematic figure of a WNL Josephson junction with the layers in the $z$-direction enumerated in the WNL and all hopping parameters indicated. Arrows indicate schematically how the spin polarization rotates in the WNL. b Band dispersion of a $n_{\mathrm{w}}=21$ layer thick WNL at zero doping $\mu=0$ along the $k_{x}$ direction, with red indicating the degenerate bands localized at the two WNL surfaces. These midgap states are degenerate since opposite spins behave the same at the two surfaces. c Spinpolarized DOS of the first and last layers (red, blue), and middle layer (yellow) of the WNL in $\mathbf{b}$.

is further dramatically enhanced thanks to the zero-energy drumhead surface states generating excellent interfaces with the $\mathrm{SCs}$. The latter effect of drumhead surface states dramatically enhancing the current we also find in DNL Josephson junctions, but there the Josephson effect is entirely conventional since there is no spin polarization. The combined effect of a huge Josephson current and odd-frequency pairing in WNLs creates what can be classified as optimal odd-frequency Josephson junctions, where an experimentally measured Josephson current becomes a direct manifestation of odd-frequency superconductivity.

\section{RESULTS}

WNL properties

We start by establishing the characteristic bulk and surface properties of WNLs. In order to clearly isolate the most important features we first use a prototype minimal model that captures the important physical details of a WNL. This is a toy model insofar as it captures an accidental nodal loop without any symmetry protection, but its simplicity significantly aids in understanding the underlying physics of WNL Josephson junctions. We then compare these results with a fully generic low-energy Hamiltonian for a WNL derived by the $k \cdot p$ method and show how the two models are in excellent agreement for the Josephson current.

A minimal WNL toy model is described by the Hamiltonian ${ }^{22,24}$ :

$H_{\mathrm{WNL}}=t_{\mathrm{w}}\left(M \sigma_{x}+2 a_{2} k_{z} \sigma_{y}\right)-\mu$,

where $M=a_{1}-k^{2}, \mathbf{k}=\left(k_{x}, k_{y}, k_{z}\right)$ with $k=|\mathbf{k}|$ is the electron wave vector, and $\sigma$ are the Pauli matrices in spin space. The equivalently minimal Hamiltonian for a DNL has instead $\sigma$ in Eq. (1) acting in orbital basis as a DNL retains spin degeneracy. Hence the minimal DNL Hamiltonian is described in the same basis as a $4 \times 4$
Hamiltonian with twofold (spin) degeneracy. Moreover, here $t_{\mathrm{w}}$ is the overall hopping amplitude, $\mu$ the chemical potential. For simplicity we measure energy in units of $t_{\mathrm{w}}$. The Fermi surface is a nodal line loop at zero doping $\mu=0$, while it forms a thin torus for nonzero $\mu$, with its shape tuned by the two parameters, $a_{1,2}$. We primarily use $a_{1}=a_{2}=1$, which results in an essentially circular Fermi surface, but our results are not sensitive to this particular choice, see Supplementary discussion. The energy dispersion away from zero energy takes the Weyl-like form $k \cdot \sigma$, giving the material class its Weyl nodal loop name ${ }^{14,23}$.

We choose to illustrate the physics of WNL Josephson junctions using the minimal Hamiltonian in Eq. (1) as it is a very simple model, offer easy comparison between WNLs and DNLs, and is also used in earlier literature discussing the immunity of the surface of WNLs toward conventional superconductivity ${ }^{24}$. Still, Eq. (1) only uses two out of the three $\sigma$-matrices, while in a generic WNL all three $\sigma$-matrices will be represented. Thus, in addition to Eq. (1), we present complementary results for a generic lowenergy Hamiltonian, which includes all three $\sigma$-matrices:

$$
H_{\mathrm{g}-\mathrm{WNL}}=t_{\mathrm{w}}\left(M \sigma_{x}+2 a_{2} k_{z}\left(k_{x}^{2}-k_{y}^{2}\right) \sigma_{y}+4 a_{2} k_{x} k_{y} k_{z} \sigma_{z}\right)-\mu
$$

which is the same low-energy Hamiltonian as proposed for the WNL $\mathrm{HgCr}_{2} \mathrm{Se}_{4}{ }^{8,10}$ upon a $90^{\circ}$ rotation of the spin axes. The generic Hamiltonian Eq. (2) breaks the $\mathcal{T} \mathcal{I}$ symmetry, i.e., the product of time-reversal $\mathcal{T}=-\mathrm{i} \sigma_{y} \mathcal{K}$, where $\mathcal{K}$ is the complex conjugation operator, and spatial inversion $\mathcal{I}:(x, y, z) \rightarrow(-x,-y,-z)$ symmetries, allowing for finite spin polarization along with spin-orbit coupling. It also contain an additional mirror symmetry, $\mathcal{M}:(x, y, z) \rightarrow(x, y,-z)$, to protect the nodal line ${ }^{8}$.

To study a finite WNL with its surface states, we place the continuum Hamiltonians Eqs. (1)-(2) on cubic lattice, performing the usual substitution of $k \rightarrow \sin (k)$ and $k^{2} \rightarrow 2(1-\cos (k))$. In the $x$ - and $y$-directions we keep the reciprocal-space description (implemented by applying periodic boundary conditions), while in the $z$ direction we discretize the Hamiltonians to produce a finite slab, see Methods. We assume a lattice with $n_{\mathrm{w}}=21$ layers along the $z$-direction, as indicated by black solid circles in Fig. 1a. Note that the distance between lattice sites are that of the full unit cell and therefore $n_{\mathrm{w}}=21$ junction length is reasonable for the Josephson effect, but we have also checked our results for much longer lengths, see Supplementary discussion.

In order to understand the basic physics of minimal WNLs, we plot in Fig. $1 \mathrm{~b}$ the energy dispersion along the $k_{x}$ direction for the Hamiltonian in Eq. (1). At zero doping the band dispersion is electron-hole symmetric and two bands, indicated in red, have a vanishing electron group velocity and form zero-energy flat bands in a large region around the $\Gamma$ point. These two bands reside on the two slab surfaces, as clearly seen in Fig. 1c, where we display the spin-polarized DOS for several different layers. The two surface layers have a very large peak at zero energy, which are fully spin polarized but in opposite directions. The bulk on the other hand has only a small constant DOS at zero energy due to the nodal line Fermi surface and there is no net spin polarization in the middle of the slab. It is the spin-orbit-like interaction term $a_{2} \sigma_{y} \sin \left(k_{z}\right)$ in the WNL Hamiltonian Eq. (1) that causes the characteristic spin rotation throughout a WNL material. This term is off-diagonal in spin space and hence couples the up and down spins in a spatially dependent manner. In a slab configuration, this term rotates the spins from spin-down polarization at zero-energy for the left surface (layer 1) to spin-up polarization for the right surface (layer $n_{\mathrm{w}}$ ), which we schematically illustrate with arrows in Fig. 1a. It is only at very large energies that the first $\left(n_{\mathrm{w}}\right.$ th) surface hosts any up- (down-)spin polarization. At finite doping the drumhead surface states remain unchanged with a full spin polarization, but are now located at an energy $\mu$ below the Fermi level. At the same time, the DOS at the Fermi level in the bulk increases due to the 


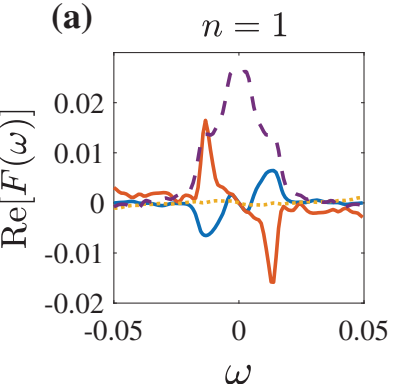

(e)

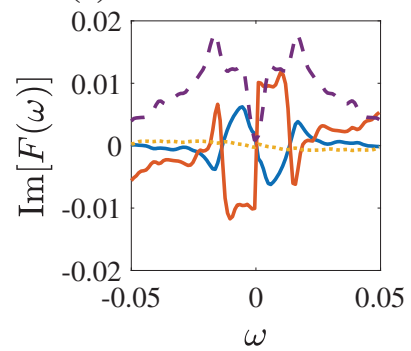

(b)

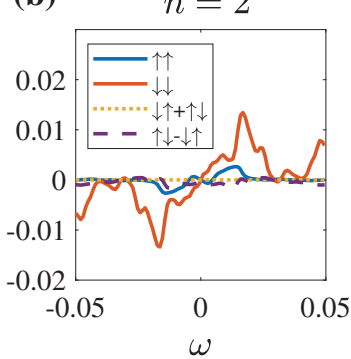

(f)

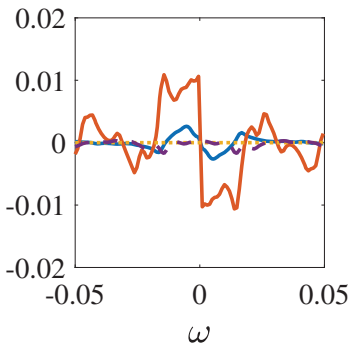

(c)

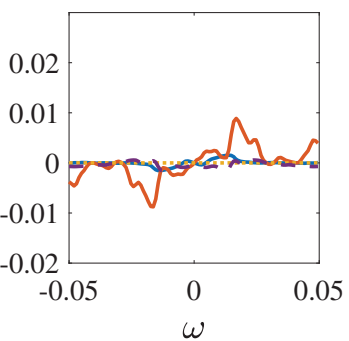

(g)

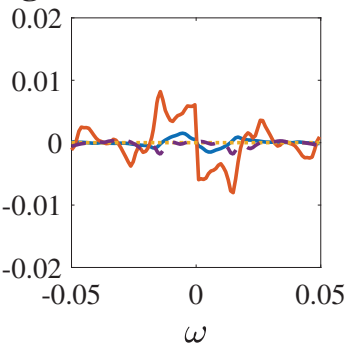

(d)

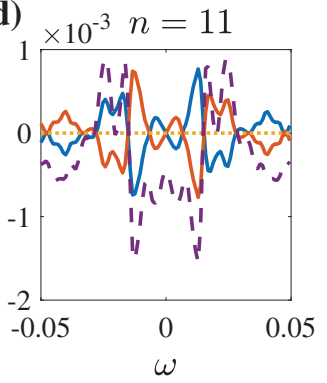

(h)

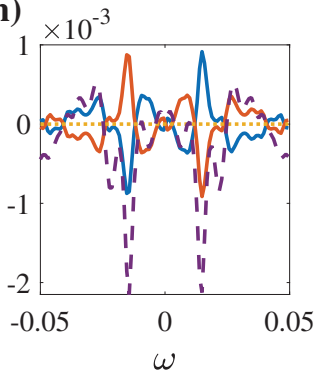

Fig. 2 Pair amplitudes in WNL Josephson junctions and their frequency dependence. Real (upper panels, a-d) and imaginary (bottom panels, e-h) parts of the anomalous Green's function $F$ as a function of frequency $\omega$, capturing the pair amplitudes divided into the equal-spin $(\uparrow \uparrow$ and $\downarrow \downarrow)$, mixed-spin $(\uparrow \downarrow+\downarrow \uparrow)$ triplet, and spin-singlet $(\uparrow \downarrow-\downarrow \uparrow)$ components. Left to right panels shows results for the $n=1,2,3$ and middle $n=\left(n_{\mathrm{w}}+1\right) / 2=11$ layers, respectively. Here we use Eq. (1) with $\mu_{\mathrm{w}}=0, t_{\mathrm{sc}-\mathrm{w}}=0.5, \Delta=0.01, t_{\mathrm{sc}}=1, \mu_{\mathrm{sc}}=2$, and $\varphi_{\mathrm{L}}=\varphi_{\mathrm{R}}=0$.

torus-shaped Fermi surface at finite doping. The spin-polarization stays however almost complete in a rather large energy window around $\mu=0$ and thus results are not sensitive to the exact tuning of the chemical potential.

\section{Odd-frequency pairing}

Next, we place two conventional spin-singlet s-wave SCs of the same superconducting material in proximity to the two surfaces of the WNL slab, see Fig. 1a. The superconducting order parameter amplitude in the SCs is set by $\Delta$, but we allow for different phases, $\varphi_{\mathrm{L}, \mathrm{R}}$ such that a Josephson current can be generated across the superconducting heterostructure. We couple the WNL and the SCs using a generic spin-independent tunneling amplitude $t_{\mathrm{sc}-\mathrm{w}} \sigma_{0}$, which connects the surface site of the left and right SCs to the left and right surfaces of the WNL, respectively.

To study proximity-induced superconductivity in the WNL we extract the superconducting pair amplitudes in the WNL by calculating the anomalous Green's function $F$ of the full heterostructure (see "Methods"). Here we only have to focus on isotropic, or equivalently on-site, s-wave pairing as we find that to be the only relevant spatial symmetry of all pair amplitudes. Notably, all the $p$-wave components in the $x y$-plane are practically zero for both WNL models; Eq. (1) even hosts an explicit in-plane spatial even parity preventing in-plane odd-parity proximity pairing. In the Supplementary discussion we additionally show that out-of-plane $p$-wave pairing is also much smaller, stemming from it not being aligned with the superconducting surface, and also that extended s-wave pairing is not relevant. Moreover, we note here that, if we were to add any disorder, that would even further favor isotropic $s$-wave pairing, thus only strengthening our results. In terms of spin configurations, the Weyl spectrum rotates the spin and can thus allow for both equal- and mixed-spin-triplet pairs. We therefore study all possible spin configurations for the superconducting pairing.

In Fig. 2 we display the real (upper panels, a-d) and imaginary (lower panels, e-h) parts of the anomalous Green's function $F$ as a function of frequency $\omega$, divided into all possible spin configurations for the minimal WNL model. Each column represent a different layer in the WNL, layers $1,2,3$, and $n=\left(n_{\mathrm{w}}+1\right) / 2$. We note directly that all different spin-triplet components appear throughout the WNL and that they are always odd functions of frequency, as required by the Fermi-Dirac statistics of the Cooper pairs. In the first surface layer there is still notable spin-singlet pairing. This is to be expected since the first layer is directly coupled to the SC and therefore necessarily harbors superconducting pairs of same symmetry as in the SC. However, the spin-singlet amplitude decay extremely quickly into the WNL, such that it has essentially disappeared already in the second layer. This behavior is not surprising when considering that the drumhead states of the WNL are fully spin polarized and thus the tunneling of opposite spins is energetically extremely costly. Similar immediate destruction of spin-singlet amplitudes have previously also been reported for $\mathrm{HM}$ junctions ${ }^{42}$. Despite the complete lack of proximity effect for spin-singlet superconductivity beyond the first surface layer, there is still significant pairing induced in the WNL. It is instead equal-spin-triplet pairing with spins aligned with the spin polarization of the drumhead state that growths and heavily dominates in the subsurface layers. Thus, the WNL essentially becomes an odd-frequency SC beyond the very first surface layer.

In the middle of the sample, Fig. $2 d$, h, all pair amplitudes are suppressed due to the distance from the SC interface, but notably, the two equal-spin pairing terms have exactly the same magnitude, just mirrored in $\omega=0$. Plotting the pair amplitudes also for the right half of the WNL, we find exactly the same results as for the left part shown in Fig. 2, only with spin-up and spindown interchanged. The behavior of the equal-spin pairing is the superconducting equivalent of the spin polarization in the normal state twisting from full spin-down polarization in the left surface layer to full spin-up polarization in the right surface layer. Thus the appearance of large odd-frequency equal-spin-triplet components in WNLs is guaranteed by the intrinsic Weyl spin-orbital structure of the WNL normal state.

To better probe the propagation of Cooper pairs inside the WNL, we plot in Fig. 3a, $b$ the absolute value of the different pair amplitudes for the minimal WNL in Eq. (1), as a function of the layer index $n$ for the whole left side of the WNL. The pair amplitudes in the right part is obtained from the left layers by just interchanging spin-up and spin-down. We display the result for 
(a)

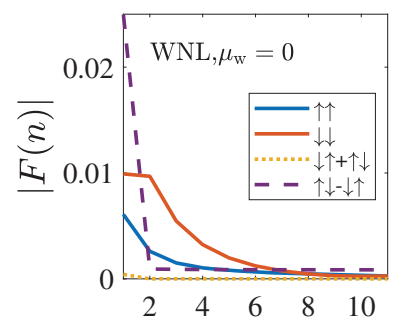

(d)

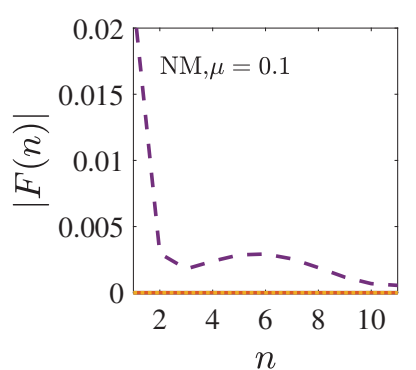

(b) $\quad \omega=0.5 \Delta$

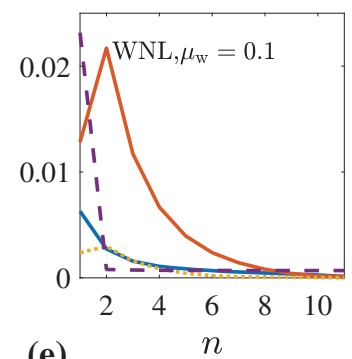

(e)

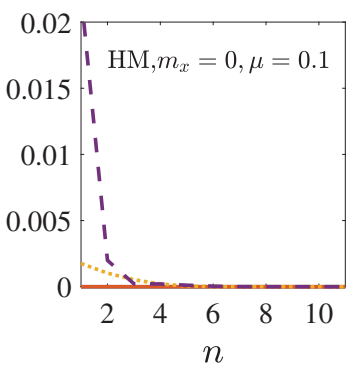

(c)

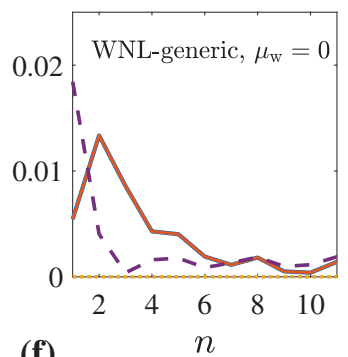

(f)

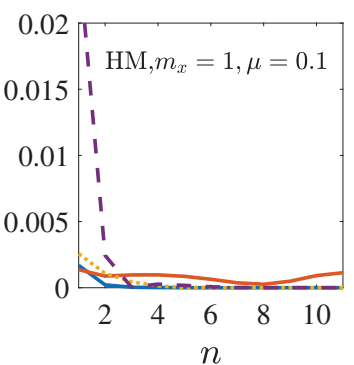

Fig. 3 Pair amplitude decay in WNL Josephson junctions compared with NM and HM junctions. Evolution of the absolute value of equalspin $(\uparrow \uparrow$ and $\downarrow \downarrow)$, mixed-spin $(\uparrow \downarrow+\downarrow \uparrow)$ triplet, and spin-singlet $(\uparrow \downarrow-\downarrow \uparrow)$ pair amplitudes into the middle of the WNL within the (a, b) minimal model Eq. (1) with chemical potential set to $\mu_{\mathrm{w}}=0,0.1$, respectively, and c generic model Eq. (2) with $\mu_{\mathrm{w}}=0$. It is compared with similar junctions with NM (d) and HM without (e) and with (f) an spin-active interface. Pair amplitudes are extracted for $\omega=0.5 \Delta$. Same parameters as in Fig. 2 for the WNL, while the NM and HM are the spin-independent part of the WNL with the HM having an additional $m_{z} \sigma_{z}$ with $m_{z}=0.5$ magnetization in the bulk and an interface $m_{x} \sigma_{z}$ with $m_{x}=1$ term modeling the spin-active interface.

two different chemical potentials, $\mu=0,0.1$, respectively, to capture both nodal loop and torus-shaped Fermi surface WNLs. Further, we set $\omega=0.5 \Delta$, but keep all other parameters as in Fig. 2 . Other choices of $\omega$ can be found in the Supplementary discussion, showing no change of trends compared with Fig. 3. We find the same extremely fast suppression of the spin-singlet amplitude for both the undoped and doped case. The mixed-spin-triplet state experiences the same decay, due to the same unfavorable spin alignment as the spin-singlet pairing. Instead, it is spin-down triplet pairing that is clearly dominating, also well into the WNL and for all $\mu$. The finite but still small spin-up triplet pair amplitude is due to probing the pair amplitudes at finite energies, where also bulk states give a finite contribution. Increasing the doping level thus show no significant changes in the relative importance of the different pairing channels. However, the magnitude of the pair amplitudes increases due to the increased bulk DOS in finite doped WNL. Overall this shows that the almost exclusively oddfrequency pairing state in the WNL is not sensitive to the tuning of the doping level. The relative insensitivity to the doping level means no doping fine-tuning is needed nor can the effects be fragile to a finite buckling of the drumhead surface state. In the Supplementary discussion we also plot the pair amplitude propagation for other lengths of the WNL junction and confirm that dominating odd-frequency pairing is also present for much longer junctions. Thus the presence of odd-frequency pairing is not just a simple surface effect, but more appropriately linked to the Weyl spin-orbital structure of the bulk band structure.

Furthermore, in Fig. 3c we present the evolution of pair amplitudes based on a generic WNL given in Eq. (2), also at $\mu=0$ to compare with panel (a). As seen, the spin-singlet pairing decays very fast, although it still have a finite amplitude in the middle of the sample. This is due to a lack of full spin polarization on the surfaces. Thus the spin-singlet response is rather similar to that of the slightly doped minimal WNL in panel (b). Dominating inside the WNL are clearly the equal-spin-triplet states, and they are even stronger in comparison to the equal-spin-triplet pairing in the minimal WNL in panel (a), which we can attribute to more strongly spin-splitted bands in the generic WNL. At the same time, the bands in the generic WNL are symmetric under interchange of spin and changing $k \rightarrow-k$, and thus both equal-spin-triplet states after summation over $k$ are equal.

To demonstrate the remarkable pairing in WNL Josephson junctions we compare the results with the behavior of similar Josephson junctions made of NM (d) and HMs without (e) and with (f) a spin-active interface region. In order to create systems with directly comparable properties we model the NM by removing all spin-dependence from the WNL Hamiltonian in Eq. (1), such that $H_{\mathrm{NM}}=t_{\mathrm{w}}\left(6-2 \cos \left(k_{x}\right)-2 \cos \left(k_{y}\right)-2 \cos \left(k_{z}\right)\right)-\mu$. This creates a prototype parabolically dispersive metal, where we set $\mu=$ 0.1 to reach a finite bulk DOS. For the NM, no term breaks the spin degeneracy, and thus only spin-singlet pairing is present in the NM. This spin-singlet amplitude experiences a regular slow decay, set by the conventional proximity effect.

For the HM junctions, we add the term $m_{z} \sigma_{z}$, with $m_{z}=0.5$, to the NM Hamiltonian $H_{\mathrm{NM}}$, such that only spin-down electrons are present at zero energy. This strong magnetization causes the same dramatic suppression of the spin-singlet amplitude as in the WNL. It also allows for spin rotation into the mixed-spin-triplet state. However, this odd-frequency spin-triplet pairing state is always small and fast-decaying, as the occurrence of spin-up components is not energetically favored by the magnetization ${ }^{44}$. To achieve a spin-down triplet component, an additional spin quantization axis has to be present in the HM junction. This is often achieved by introducing a spin-active region at the interface between the SC and HM, see e.g., ${ }^{42,43}$. In this case the Cooper pair spin quantization axis rotates between the interface and the HM bulk, with the consequence that spin-equal pairing is induced beyond the interface. In Fig. $3 f$ we therefore add a term $m_{x} \sigma_{x}$, with $m_{x}=1$, to the the two surface layers of the HM to model a strongly spinactive interface. As a result, spin-equal triplet pairing is generated, of which the spin-down component survives throughout the HM region. The spin-up triplet state is also initially generated at the interface, but it is energetically unfavorable and decays very quickly in the HM. 
(a)

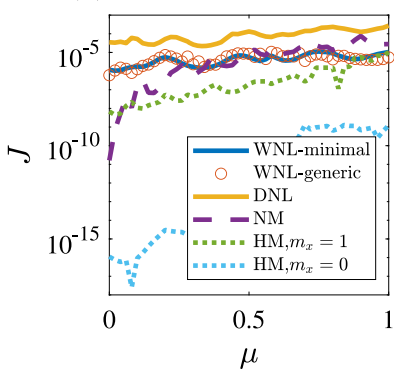

(b)

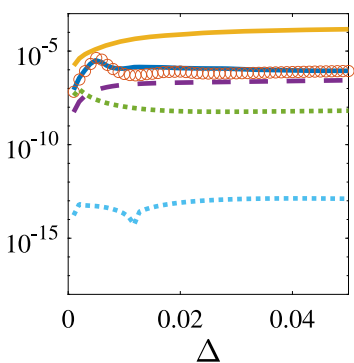

(c)

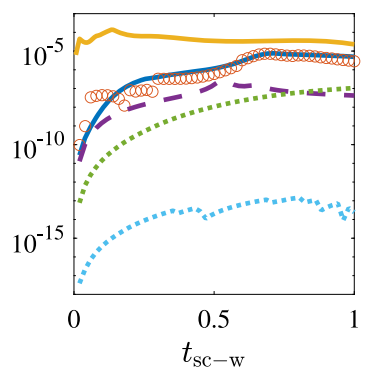

Fig. 4 Josephson current in WNL Josephson junctions compared with DNL, NM, and HM junctions. Parameter dependencies of the Josephson current (in units of e $a^{2} t_{\mathrm{w}} \hbar^{-1}$ ) in WNL, DNL, NM, and HM Josephson junctions as a function of chemical potential $\mu$ (a), order parameter amplitude $\Delta(\mathbf{b})$, and tunneling between the SC and junction material $t_{\text {sc-w }}$ (c). Fixed parameters are the same as in Figs. 2 and 3 except $\varphi_{\mathrm{L}}=\pi / 2, \varphi_{\mathrm{R}}=0$ and in $\mathbf{b}, \mathbf{c}$ NM and HM have $\mu=0.1$, while $\mu_{\mathrm{w}}=0$ in the WNL.

Comparing the WNLs with the NM and HM junctions, we see that the WNLs junction closest resemble that of the HM with an active spin interface, since they both experience a strong proximity effect consisting of odd-frequency equal-spin-triplet pairing. However, WNL Josephson junctions are fundamentally different from HM Josephson junctions as they do not need any additional spin-active interface region added during manufacturing in order to generate equal-spin odd-frequency pairing. In the simplistic WNL junctions based on Eq. (1) we find that the intrinsic Weyl spin-orbit coupling causes the initial spin-down pairing in the left surface to rotate into spin-up pairing in the right surface. As a consequence, there is a clear decay of the spin-up triplet component into the middle of the WNL, which is not present in the HM. It is thus not the distance from the SC that causes the main decay of the equal-spin-triplet components in Fig. 3a, b, but mainly the continuous rotation of the spin orientation of the Cooper pairs. This is also clear when considering other WNL junctions lengths in the Supplementary discussion. For the generic WNL model, we find that both equal-spin-triplet pairing are present at equal amounts in each layer due to its spin structure, and then there is a smaller decay of the odd-frequency state away from the surface of the WNL.

We also note that the size of the pair amplitudes in the WNLs at zero doping is actually of the same order of magnitude as in the NM junctions. This is particularly surprising since the nodal line bulk state has a much smaller DOS at low energies compared with the NM (see Supplementary discussion for a detailed account on the low-energy DOS). We attribute the large pair amplitudes in undoped WNLs to the singular zero-energy DOS of the drumhead surface states, which creates a naturally strong coupling between the WNL and SCs. The large effect of the surface drumhead states is further evident when we compare the results for a DNL Josephson junction. Here only spin-singlet s-wave pairing is present since the DNL Hamiltonian is spin degenerate, but we find a very large conventional proximity effect due to the the (unpolarized) surface drumhead states, see Supplementary discussion for details.

\section{Exotic Josephson current}

Having shown how odd-frequency spin-triplet pairing generates large proximity-induced superconductivity in WNLs, despite the incompatibility of spin-polarization and the spin-singlet SCs, we now turn to the possibility of measuring a finite Josephson current in the WNL junctions. For comparison we also calculate the current in DNL, NM, and HM Josephson junctions. While we limited the investigation in Figs. 2-3 to isotropic s-wave pairing, the contributions from all pairing channels are automatically included when calculating the current, although all non-s-wave contributions are small for WNLs.
In Fig. 4 we show in a log-plot the maximum Josephson current $J$ between two conventional spin-singlet SCs as a function of the chemical potential $\mu$, superconducting order parameter $\Delta$, and WNL-SC tunneling $t_{\mathrm{sc}-\mathrm{w}}$. The results are obtained for $\varphi_{\mathrm{L}}=\pi / 2, \varphi_{\mathrm{R}}=$ 0 , which to a very good approximation gives the maximum current as $J \sim \sin \left(\varphi_{L}-\varphi_{R}\right)$, see Supplementary discussion. The most remarkable result is that WNL junctions carry a large Josephson current through all parameter regimes, orders of magnitude higher than the HM junctions, the other odd-frequency systems, but also compared with the NM junctions for a large range of doping levels. The current for both WNL junctions is also always essentially identical, illustrating explicitly that the specific form of the WNL Hamiltonian is irrelevant for the Josephson current. We note particularly that the slightly different spin-singlet pairing in the two different WNL junctions do not influence the Josephson effect. This can also be understood from the results for NM junction, which only contains spin-singlet pairing but where the Josephson current is orders of magnitude smaller than the WNL junctions in the small doping regime. Instead it is the common physical properties of WNLs, their strong spinpolarization (surface and bulk), and drumhead surface states, that generates a unique odd-frequency system with large Josephson currents. WNL Josephson effect thus forms a key example on the importance of odd-frequency pairing in inhomogeneous superconducting systems. If it were not for the odd-frequency correlations, there would be no proximity-induced superconductivity or measurable Josephson effect in WNL junctions.

In particular, we find for increasing $\mu$ in Fig. 4a that the current increases for all junctions, while also displaying some overlaid oscillatory behavior. This is expected since the low-energy DOS increases with $\mu$ for all junctions with some smaller oscillations due to finite size effects. Most notably, the WNL junctions carry a very large current over a wide range of low to moderate doping levels, even orders of magnitude larger than both the NM and HM junctions. This is really quite exceptional considering the low bulk DOS of the nodal line/thin torus Fermi surface in the bulk of WNLs. In fact, the WNL bulk DOS for Eq. (1) is for the full doping range smaller than that of the bulk NM, for $\mu \lesssim 0.4$ it is even several times smaller, see Supplementary discussion. We must therefore accredit the large Josephson current in WNL junctions to a remarkable effect of the drumhead surface states.

The effect of the drumhead states is even more obvious when we compare to a DNL Josephson junction. Here we use a DNL with the same band structure and total DOS as the WNL in Eq. (1), but keep a fully spin-degenerate ground state. Thus the Josephson effect and current are entirely conventional and carried by evenfrequency spin-singlet s-wave pairs in a DNL, while the coupling to the SCs is determined by the same surface DOS peak as in the WNL junction. We find that the DNL junction carries a current that is consistently large overall parameter regimes investigated in Fig. 4. In the simplest of cases, the maximum Josephson current in a 
junction is proportional to the normal current, which in turn is proportional to $\mathrm{e}^{2} \rho v_{\mathrm{F}}$, where $\rho$ is the DOS and $v_{\mathrm{F}}$ is the Fermi velocity in the direction of the current. While the DOS varies within the junction for both DNLs and WNLs, this still demonstrates that the drumhead surface state should dramatically enhance the current in both cases. We here note that in general flat band systems the current usually suffers from the diminishing Fermi velocity. However, for the DNL and WNL junctions considered here it is only the Fermi velocity in-plane that is quenched due to the flat dispersion, while it is the velocity in the out-of-plane direction that determine the current, which is still large. We also note that the DNL current is larger than the WNL current, which we can easily attribute to the DNL junction only hosting conventional pairing and thus does not have to transform to other pair amplitude symmetries to create a viable Josephson effect.

Moving on to compare with the HM junctions that also host odd-frequency spin-triplet pairs, we find that the HM without a spin-active interface cannot effectively carry Josephson current, which is a direct consequence of the lack superconducting pair amplitudes in the HM. Introducing a spin-active interface layer, we find a significantly increased current due to the perseverance of odd-frequency equal-spin-triplet pairs inside the HM. Yet, it is only at extremely large $\mu$ that the HM system carries a similarly sized current to the WNL junction. For large $\mu$ the drumhead surface states are located very far from the Fermi level and are thus less active in electric transport. Thus, the large current in heavily doped WNL junctions is instead primarily a manifestation of how powerful the Weyl spin-orbit interaction is in generating oddfrequency equal-spin pairing to carry the current, and thus it is natural that the WNL and HM junction behave similarly in this extreme doping limit.

We here note that enhancement of Josephson current in junctions with odd-frequency pairing has previously been associated with generation of zero-energy Andreev bound states, as for example in SC-insulator-SC junctions ${ }^{45-48}$. In fact, zeroenergy Andreev bound states have been found to be very common in systems with odd-frequency pairing ${ }^{49-51}$, although not always present ${ }^{52-55}$. In the Supplementary discussion we show that zero-energy Andreev boundary states also exists at the WNL$\mathrm{SC}$ interface. However, considering the much larger current for the WNL junction as compared with the HM junctions, zero-energy Andreev bound states alone cannot alone explain the results, but instead the drumhead surface states are more important.

Finally, turning Fig. 4b, $c$, we explore the other parameter dependencies of the Josephson current. Again note that both models of WNL are in a excellent agreement with each other, and thus our main conclusions are not dependent on any specific model. Figure $4 \mathrm{~b}$ shows the variation of the Josephson current with respect to the superconducting gap parameter $\Delta$, keeping the chemical potential of NM and HM at $\mu=0.1$, while the WNLs are both undoped. Thus, both the NM and HM have a large metallic DOS at low energies, while the DNL and WNL have only 1D Fermi nodal loops. Despite this we see how the Josephson current is actually larger in WNL junctions compared with the NM and $\mathrm{HM}$ junctions for all $\Delta$ values. Notably, the choice of $\Delta=0.01$ in Figs. 2 and 3 gives by no means the maximum Josephson current, for example choosing $\Delta \approx 0.005$ gives approximately three times larger current. In Fig. $4 c$ we then tune the tunneling between the SCs and the junction, $t_{\mathrm{sc}-\mathrm{w}}$. Usually this parameter is lower than the hopping inside the junction and in the SCs, limiting it to $t_{\mathrm{sc}-\mathrm{w}}<1$, and in practice it is tuned by modifying the interfaces. Larger tunneling clearly enhances the Josephson current in all junctions, but we find that the WNL junctions again carry larger Josephson current than the NM and HM junctions for all values of $t_{\text {sc-w. }}$. In particular, a large Josephson current is present even for small $t_{\mathrm{sc}-\mathrm{w}} \sim 0.1$, where the overall effect of the SCs on the electronic structure of the WNL is necessarily quite limited. We provide additional plots for other choices of chemical potential in
NM and HM in the Supplementary discussion, verifying that the large Josephson currents through the WNL junctions in Fig. 4 are generic results and not restricted to a narrow range of physical parameters.

\section{DISCUSSION}

To summarize we establish a large and exotic Josephson effect in WNL superconducting junctions, driven by spin-polarized surface and bulk states and bulk Weyl spin-orbit interaction. Combined these effects enable a very effective transformation of the original spin-singlet pairing in the SC leads into an odd-frequency equalspin-triplet pairing state, which then carries the Josephson current into the WNL. Further, the singular DOS of the drumhead surface states make carrier transport between the SCs and the WNL very effective, which significantly enhances the current. In fact, we find that the WNL Josephson current can easily be orders of magnitude larger than the current in NM Josephson junctions, despite a much lower bulk DOS in the WNL due to its nodal line Fermi surface. A similar huge Josephson current also exists for DNL due to its equivalently large surface DOS peak, but here the current is carried by conventional spin-singlet superconductivity and is thus an expected result. Notably we find exceptionally similar Josephson currents in both simple and generic models for WNLs. Thus our results are not sensitive to the particular choice of model for the WNL but depend on the general features of WNLs.

The physics of the WNL Josephson junction can be understood from the behavior of $\mathrm{HM}$ junctions with spin-active interfaces, as both junctions have dominating odd-frequency equal-spin-triplet pairing carrying the Josephson current. However, the strong bulk spin-orbit coupling in the WNL Josephson junction make it more similar to HM junctions with a helical magnet configuration instead of just a spin-active interface. Such helical magnets have recently become the prototype experimental odd-frequency system due to their large and dominating odd-frequency response probed by both Josephson effect and paramagnetic Meissner effect $^{37,56-58}$. Still, the WNL is a much more optimal odd-frequency Josephson link due to the drumhead surface states allowing for excellent interfacial coupling and thus dramatically larger currents. In fact, this huge Josephson current would not even have existed if it was not for odd-frequency superconductivity. The importance of the drumhead surface states persists as long as the junction is not much longer than the decay length of the conventional superconducting proximity effect, as then most pairing occurring in the surface states still propagate through the junction. Thus the WNL odd-frequency Josephson current exists on the same length scales as conventional Josephson effect. In conclusion, the combination of finite spin-polarization, Weyl spin-orbit interaction, and drumhead surface states makes WNLs optimal oddfrequency materials, with the odd-frequency pairing detectable by finite and unexpectedly large Josephson currents.

\section{METHODS}

For the WNL we consider a finite slab in the $z$-direction. We use the reciprocal-space continuum Hamiltonian in Eq. (1) for $\left(k_{x}, k_{y}\right)=\mathbf{k}_{\|}$, while discretizing the model in the $z$-direction in the standard way: $t_{\mathrm{w}} \cos \left(k_{z}\right)$ becomes the nearest neighbor hopping $t_{\mathrm{w}}$, while $t_{\mathrm{w}} \sigma_{y} \sin \left(k_{z}\right)$ generates a nearest neighbor spin-orbit interaction. Moreover, in the $x, y$-directions we perform the substitution $k \rightarrow \sin (k)$ and $k^{2} \rightarrow 2(1-\cos (k))$, to comply with a lattice description. For concreteness we use $n_{\mathrm{w}}=21$ layers in the $z$ direction, but our results are not sensitive to the number of layers, granted that the surface states are spatially well separated, and the junction still carry a supercurrent for a NM. By calculating the retarded Green's function $G=\left(\omega+\mathrm{iO}^{+}-H\right)^{-1}$ we obtain the spin-resolved DOS in each WNL layer $n$ as $\rho_{n, \sigma}=-\frac{1}{\pi} \Im \sum_{\mathbf{k}_{\|}} G_{n, \sigma}\left(\mathbf{k}_{\|}, \omega+\mathrm{i} 0^{+}\right)$. 
To investigate Josephson junctions we attach a conventional SC to each of the two WNL slab surfaces with the Hamiltonian in Nambu space being

$\mathcal{H}_{s c}^{j}=\left(\begin{array}{cc}h_{s c} \sigma_{0} & \Delta \mathrm{e}^{\mathrm{i} \varphi_{j}} \sigma_{y} \\ \Delta \mathrm{e}^{-\mathrm{i} \varphi_{j}} \sigma_{y} & -h_{\mathrm{sc}} \sigma_{0}\end{array}\right)$,

where $j=\mathrm{L}, \mathrm{R}$ indicates the left and right SC, respectively. We define the $\mathrm{SCS}$ on a simple cubic lattice, where the normal state Hamiltonian in $\left(k_{x}, k_{y}\right)$ takes the form $h_{\mathrm{sc}}=t_{\mathrm{sc}}\left(2-\cos \left(k_{x} a\right)-\cos \left(k_{y} a\right)\right)-\mu_{\mathrm{sc}}$ due to translational invariance, while in the $z$-direction we have the nearest neighbor hopping $t_{\mathrm{sc}}$ between the different layers of the SCs. Spin-singlet $s$-wave superconductivity is implemented as usual by an on-site order parameter $\Delta \mathrm{e}^{\mathrm{i} \varphi_{j}}$. We use $n_{\mathrm{sc}}=20$ layers for each SC and set $t_{\mathrm{sc}}=1$ and $\mu_{\mathrm{sc}}=2$ to create SCs with large low-energy DOS. The WNL and SCs are connected by a generic spin-independent tunneling $t_{s c-w}$. The results are not qualitatively sensitive to $n_{\mathrm{sc}}$ or other physical parameters in the SCs, as shown in the Supplementary discussion. As a result, the total Hamiltonian takes the form

$\mathcal{H}_{t}=\left(\begin{array}{ccc}\mathcal{H}_{\mathrm{SC}}^{\mathrm{L}} & T_{\mathrm{L}} & 0 \\ T_{\mathrm{L}}^{\dagger} & \mathcal{H}_{\mathrm{W}} & T_{\mathrm{R}}^{\dagger} \\ 0 & T_{\mathrm{R}} & \mathcal{H}_{\mathrm{SC}}^{\mathrm{R}}\end{array}\right)$,

where $\mathcal{H}_{\mathrm{W}}$ is the Nambu representation of the WNL Hamiltonian in Eqs. (1) or (2). Moreover, the matrices $T_{\mathrm{L} / \mathrm{R}}$ are $4 n_{\mathrm{sc}} \times 4 n_{\mathrm{w}}$ matrices that connects the corresponding surfaces of the WNL to the left and right SCs.

Using the same Green's function technique but now the whole system $\mathrm{WNL}+\mathrm{SCs}$, we extract the anomalous electron-hole part, which is proportional to $\left\langle c_{n \sigma}^{\dagger} c_{n \sigma^{\prime}}^{\dagger}\right\rangle$, with $c_{n \sigma}^{\dagger}$ the electron creation operator in layer $n$ with spin $\sigma$, and thus gives the pair correlations in all SC and WNL layers. By integrating over $\mathbf{k}_{\|}$we obtain the $s$-wave contribution, which we report individually for each pair spin configuration. Note that in order to achieve the correct pair amplitudes as a function of $\omega$, we need to use the advanced (retarded) Green's function for negative (positive) frequencies ${ }^{27,59}$.

To calculate the Josephson current between two SCs, we use the continuity equation $\nabla \cdot \mathbf{J}+\left\langle\partial \hat{\rho}_{n} / \partial t\right\rangle=0$, where $\mathbf{J}$ is the current density vector and $\hat{\rho}_{n}=\sum_{\sigma} c_{n \sigma}^{\dagger} c_{n \sigma}$ is the density operator ${ }^{60,61}$. Since we are working only with layers in the $z$-direction, we sum overall $\mathbf{k}_{\|}$. Moreover, $\langle\ldots\rangle$ indicates the expectation value taken over whole system, which we obtain by summing overall occupied energy eigenstates. Finally, the derivative of the density operator $\hat{\rho}_{n}$ can be obtained from $\left\langle\partial \hat{\rho}_{n} / \partial t\right\rangle=\left\langle\left[H, \hat{\rho}_{n}\right]\right\rangle$. Here the right-hand side generates terms of the type, $c_{n}^{\dagger} c_{n+1}$ and $c_{n}^{\dagger} c_{n-1}$, which are intuitively proportional to the in- and out-going currents $\left(j_{\text {inn }}, j_{\text {out }}\right)$ in each layer. Writing $\nabla \cdot \mathbf{J}=\left(j_{\text {out }}-j_{\text {in }}\right) / a^{2}$, we can obtain the Josephson current $J=\mathrm{e}\left(j_{\text {out }}-j_{\text {in }}\right)$ in units of $e a^{2} t_{\mathrm{w}} \hbar^{-1}$.

\section{DATA AVAILABILITY}

The datasets generated during the current study are available from the corresponding author on reasonable request.

Received: 23 May 2019; Accepted: 4 June 2020; Published online: 25 June 2020

\section{REFERENCES}

1. Yang, S.-Y. et al. Symmetry demanded topological nodal-line materials. Adv. Phys. $X$ 3, 1414631 (2018).

2. Gao, H., Venderbos, J. W. F., Kim, Y. \& Rappe, A. M. Topological semimetals from first-principles. Annu. Rev. Mater. Res. 49, 153-183 (2019).

3. Chen, Y., Lu, Y.-M. \& Kee, H.-Y. Topological crystalline metal in orthorhombic perovskite iridates. Nat. Commun. 6, 6593 (2015).

4. Schaffer, R., Lee, E. K.-H., Lu, Y.-M. \& Kim, Y. B. Topological spinon semimetals and gapless boundary states in three dimensions. Phys. Rev. Lett. 114, 116803 (2015).

5. Kim, Y., Wieder, B. J., Kane, C. L. \& Rappe, A. M. Dirac line nodes in inversionsymmetric crystals. Phys. Rev. Lett. 115, 036806 (2015).

6. Yu, R. et al. Topological node-line semimetal and Dirac semimetal state in antiperovskite $\mathrm{Cu}_{3}$ PdN. Phys. Rev. Lett. 115, 036807 (2015)

7. Bouhon, A. \& Black-Schaffer, A. M. M. Bulk topology of line-nodal structures protected by space group symmetries in class Al. Preprint at https://arxiv.org/abs/ 1710.04871 (2017).

8. Xu, G. et al. Semimetal and the quantized anomalous Hall effect in $\mathrm{HgCr}_{2} \mathrm{Se}_{4}$. Phys. Rev. Lett. 107, 186806 (2011).
9. Sun, X.-Q., Zhang, S.-C. \& Bzdušek, T. Conversion rules for Weyl points and nodal lines in topological media. Phys. Rev. Lett. 121, 106402 (2018).

10. Chen, W., Luo, K., Li, L. \& Zilbergerg, O. Proposal for detecting nodal-line semimetal surface states with resonant spin-flipped reflection. Phys. Rev. Lett. 121, 166802 (2018).

11. Fang, C., Weng, H., Dai, X. \& Fang, Z. Topological nodal line semimetals. Chin. Phys. B 25, 117106 (2016).

12. Bian, G. et al. Topological nodal-line Fermions in spin-orbit metal PbTaSe $\mathrm{Pat}_{2}$. Commun. 7, 10556 (2016).

13. Schoop, L. M. et al. Dirac cone protected by non-symmorphic symmetry and three-dimensional Dirac line node in ZrSiS. Nat. Commun. 7, 11696 (2016).

14. Xie, L. S. et al. A new form of $\mathrm{Ca}_{3} \mathrm{P}_{2}$ with a ring of Dirac nodes. APL Mater. 3, 083602 (2015)

15. Takane, D. et al. Observation of Dirac-like energy band and ring-torus Fermi surface associated with the nodal line in topological insulator CaAgAs. npj Quantum Mater. 3, 1 (2018).

16. Bzdusek, T. et al. Nodal-chain metals. Nature 538, 75-78 (2016).

17. Weng, $H$. et al. Topological node-line semimetal in three-dimensional graphene networks. Phys. Rev. B 92, 045108 (2015).

18. Pezzini, S. et al. Unconventional mass enhancement around the Dirac nodal loop in ZrSiS. Nat. Phys. 14, 178-183 (2017).

19. Kopnin, N. B., Heikkilä, T. T. \& Volovik, G. E. High-temperature surface superconductivity in topological flat-band systems. Phys. Rev. B 83, 220503 (2011).

20. Heikkilä, T. T., Kopnin, N. B. \& Volovik, G. E. Flat bands in topological media. JETP Lett. 94, 233 (2011).

21. Löthman, T. \& Black-Schaffer, A. M. Universal phase diagrams with superconducting domes for electronic flat bands. Phys. Rev. B 96, 064505 (2017).

22. Nandkishore, R. Weyl and Dirac loop superconductors. Phys. Rev. B 93, 020506 (2016).

23. Sur, S. \& Nandkishore, R. Instabilities of Weyl loop semimetals. N. J. Phys. 18, 115006 (2016).

24. Wang, Y. \& Nandkishore, R. M. Topological surface superconductivity in doped Weyl loop materials. Phys. Rev. B 95, 060506 (2017).

25. Berezinskii, V. L. New model of the anisotropic phase of superfluid $\mathrm{He}^{3}$. Pisma Zh. Eksp. Teor. Fiz. 20, 628-631 (1974).

26. Bergeret, F. S., Volkov, A. F. \& Efetov, K. B. Odd triplet superconductivity and related phenomena in superconductor-ferromagnet structures. Rev. Mod. Phys. 77, 1321-1373 (2005).

27. Linder, J. \& Balatsky, A. V. Odd-frequency superconductivity. Rev. Mod. Phys. 91, 045005 (2019)

28. Keizer, R. S. et al. A spin triplet supercurrent through the half-metallic ferromagnet $\mathrm{CrO}_{2}$. Nature 439, 825-827 (2006).

29. Bergeret, F. S., Volkov, A. F. \& Efetov, K. B. Long-range proximity effects in superconductor-ferromagnet structures. Phys. Rev. Lett. 86, 4096-4099 (2001).

30. Tanaka, Y., Asano, Y., Golubov, A. A. \& Kashiwaya, S. Anomalous features of the proximity effect in triplet superconductors. Phys. Rev. B 72, 140503 (2005).

31. Black-Schaffer, A. M. \& Balatsky, A. V. Odd-frequency superconducting pairing in multiband superconductors. Phys. Rev. B 88, 104514 (2013).

32. Triola, C. \& Balatsky, A. V. Pair symmetry conversion in driven multiband superconductors. Phys. Rev. B 95, 224518 (2017).

33. Abrahams, E., Balatsky, A., Scalapino, D. J. \& Schrieffer, J. R. Properties of odd-gap superconductors. Phys. Rev. B 52, 1271-1278 (1995).

34. Yokoyama, T., Tanaka, Y. \& Nagaosa, N. Anomalous Meissner effect in a normalmetal-superconductor junction with a spin-active interface. Phys. Rev. Lett. 106, 246601 (2011).

35. Asano, Y., Golubov, A. A., Fominov, Y. V. \& Tanaka, Y. Unconventional surface impedance of a normal-metal film covering a spin-triplet superconductor due to odd-frequency Cooper pairs. Phys. Rev. Lett. 107, 087001 (2011).

36. Asano, Y. \& Sasaki, A. Odd-frequency Cooper pairs in two-band superconductors and their magnetic response. Phys. Rev. B 92, 224508 (2015).

37. Di Bernardo, A. et al. Intrinsic paramagnetic Meissner effect due to $s$-wave oddfrequency superconductivity. Phys. Rev. X 5, 041021 (2015).

38. Alidoust, M., Halterman, K. \& Linder, J. Meissner effect probing of oddfrequency triplet pairing in superconducting spin valves. Phys. Rev. B 89, 054508 (2014).

39. Komendova, L. \& Black-Schaffer, A. M. Odd-frequency superconductivity in $\mathrm{Sr}_{2}$ $\mathrm{RuO}_{4}$ measured by Kerr rotation. Phys. Rev. Lett. 119, 087001 (2017).

40. Triola, C. \& Black-Schaffer, A. M. Odd-frequency pairing and Kerr effect in the heavy-fermion superconductor UPt ${ }_{3}$. Phys. Rev. B 97, 064505 (2018).

41. Eschrig, M., Kopu, J., Cuevas, J. C. \& Schon, G. Theory of half-metal/superconductor heterostructures. Phys. Rev. Lett. 90, 137003 (2003).

42. Asano, Y., Tanaka, Y. \& Golubov, A. A. Josephson effect due to odd-frequency pairs in diffusive half metals. Phys. Rev. Lett. 98, 107002 (2007).

43. Eschrig, M. \& Löfwander, T. Triplet supercurrents in clean and disordered halfmetallic ferromagnets. Nat. Phys. 4, 138-143 (2008). 
44. Parhizgar, F. \& Black-Schaffer, A. M. Unconventional proximity-induced superconductivity in bilayer systems. Phys. Rev. B 90, 184517 (2014).

45. Tanaka, Y. \& Kashiwaya, S. Theory of the Josephson effect in $d$-wave superconductors. Phys. Rev. B 53, R11957-R11960 (1996).

46. Barash, Y. S., Burkhardt, H. \& Rainer, D. Low-temperature anomaly in the Josephson critical current of junctions in $d$-wave superconductors. Phys. Rev. Lett. 77, 4070-4073 (1996).

47. Tanaka, Y. \& Kashiwaya, S. Theory of Josephson effects in anisotropic superconductors. Phys. Rev. B 56, 892-912 (1997).

48. Kwon, H.-J., Sengupta, K. \& Yakovenko, V. M. Fractional ac Josephson effect in $p$ and $d$-wave superconductors. Eur. Phys. J. B 37, 349-361 (2004).

49. Tanaka, Y., Tanuma, Y. \& Golubov, A. A. Odd-frequency pairing in normal-metal/ superconductor junctions. Phys. Rev. B 76, 054522 (2007).

50. Tanaka, Y., Golubov, A. A., Kashiwaya, S. \& Ueda, M. Anomalous Josephson effect between even- and odd-Frequency Superconductors. Phys. Rev. Lett. 99, 037005 (2007).

51. Tanaka, Y., Sato, M. \& Nagaosa, N. Symmetry and topology in superconductors 'odd-frequency pairing and edge states. J. Phys. Soc. Jpn. 81, 011013 (2012).

52. Robinson, J. W. A. \& Linder, J. Strong odd-frequency correlations in fully gapped Zeeman-split superconductors. Sci. Rep. 5, 15483 (2015).

53. Black-Schaffer, A. M. \& Balatsky, A. V. Odd-frequency superconducting pairing in topological insulators. Phys. Rev. B 86, 144506 (2012).

54. Black-Schaffer, A. M. \& Balatsky, A. V. Proximity-induced unconventional superconductivity in topological insulators. Phys. Rev. B 87, 220506(R) (2013).

55. Black-Schaffer, A. M. \& Balatsky, A. V. Odd-frequency superconducting pairing in multiband superconductors. Phys. Rev. B 88, 104514 (2013).

56. Robinson, J. W. A., Witt, J. D. S. \& Blamire, M. G. Controlled injection of spin-triplet supercurrents into a strong ferromagnet. Science 329, 59-61 (2010).

57. Linder, J. \& Robinson, J. W. A. Superconducting spintronics. Nat. Phys. 11, 307-315 (2015).

58. Di Bernardo, A. et al. Signature of magnetic-dependent gapless odd frequency states at superconductor/ferromagnet interfaces. Nat. Commun. 6, 8053 (2015).

59. Cayao, J. \& Black-Schaffer, A. M. Odd-frequency superconducting pairing and subgap density of states at the edge of a two-dimensional topological insulator without magnetism. Phys. Rev. B 96, 155426 (2017).

60. Black-Schaffer, A. M. \& Doniach, S. Self-consistent solution for proximity effect and Josephson current in ballistic graphene SNS Josephson junctions. Phys. Rev. B 78, 024504 (2008).

61. Björnson, K., Pershoguba, S. S., Balatsky, A. V. \& Black-Schaffer, A. M. Spinpolarized edge currents and Majorana fermions in one- and two-dimensional topological superconductors. Phys. Rev. B 92, 214501 (2015).

\section{ACKNOWLEDGEMENTS}

We thank A. Balatsky, K. Björnson, J. Cayao, P. Dutta, S. Nahas, and C. Triola for fruitful discussions and acknowledge financial support from the Swedish Research Council
(Vetenskapsrå det, Grant No. 621-2014-3721), the Göran Gustafsson Foundation, the Wallenberg Academy Fellows program through the Knut and Alice Wallenberg Foundation, and the European Research Council (ERC) under the European Unions Horizon 2020 research and innovation program (ERC-2017-StG-757553). Open access funding provided by Uppsala University.

\section{AUTHOR CONTRIBUTIONS}

F.P. carried out the calculations and both authors analyzing the results. A.B.S. provided the initial idea, while the authors wrote the paper together.

\section{COMPETING INTERESTS}

The authors declare no competing interests.

\section{ADDITIONAL INFORMATION}

Supplementary information is available for this paper at https://doi.org/10.1038/ s41535-020-0244-2.

Correspondence and requests for materials should be addressed to F.P. or A.M.B.-S.

Reprints and permission information is available at http://www.nature.com/ reprints

Publisher's note Springer Nature remains neutral with regard to jurisdictional claims in published maps and institutional affiliations.

cC (i) Open Access This article is licensed under a Creative Commons (c) Attribution 4.0 International License, which permits use, sharing, adaptation, distribution and reproduction in any medium or format, as long as you give appropriate credit to the original author(s) and the source, provide a link to the Creative Commons license, and indicate if changes were made. The images or other third party material in this article are included in the article's Creative Commons license, unless indicated otherwise in a credit line to the material. If material is not included in the article's Creative Commons license and your intended use is not permitted by statutory regulation or exceeds the permitted use, you will need to obtain permission directly from the copyright holder. To view a copy of this license, visit http://creativecommons. org/licenses/by/4.0/.

(c) The Author(s) 2020 